\title{
2-day, 3-day, and 5-6-day oscillations of the geomagnetic field detected by principal component analysis
}

\author{
Yuji Yamada \\ Kakioka Magnetic Observatory, 595 Kakioka, Yasato-machi, Niihari-gun, Ibaraki 315-0116, Japan
}

(Received January 16, 2001; Revised December 27, 2001; Accepted January 8, 2002)

\begin{abstract}
The day-to-day variation of the geomagnetic daily variation has been examined using hourly data of the $Z$ component at Chichijima (geographic $27.15^{\circ} \mathrm{N}, 142.30^{\circ} \mathrm{E}$; geomagnetic $18.11^{\circ} \mathrm{N}$ ) in the northwestern Pacific Ocean. Principal component analysis is applied to the data from 1970 to 1992, and 2-day, 3-day, and 5-6-day oscillations were extracted from the day-to-day variation of the daily profile. The temporal variations of both the waveform and amplitude of each oscillation were clarified. The fluctuation associated with any of these oscillations is confined to the sunlit hours, which infers the oscillations are originated from ionospheric currents. Although the amplitudes of the oscillations are variable, they seem to be present almost continuously. Each oscillation is expressed primarily by the periodic increase and decrease of the amplitude of the quiet time daily variation, and the deviation from the normal level statistically amounts to $12 \%$ (3-day oscillation), 15\% (5-6-day oscillation), and $17 \%$ (2-day oscillation) of the mean amplitude of the daily variation. There are cases, however, for which the magnitude is considerably amplified during a short duration. There also exist the oscillations that show the three periods in the pattern of the daily variation as well as in intensity. The result of analysis of day-to-day variations at other four stations indicates that the oscillations are phenomena having some latitudinal extent across the geomagnetic equator.
\end{abstract}

\section{Introduction}

The day-to-day variation of the geomagnetic solar quiet daily variation $(S q)$ has been investigated by many workers. One of the origins of the day-to-day variation is probably a fluctuation of a tidal wind because the daily variation is attributed mainly to the ionospheric currents driven by the wind. While Phillips and Briggs (1991) found no correlation of the daily magnetic field strength with the diurnal and semi-diurnal tidal winds in the lower thermosphere, Kohsiek et al. (1998) detected a signature of the day-to-day coupling between the tidal wind and the $S q$ magnetic field in the middle latitudes. On the other hand, some workers have examined planetary waves in the atmosphere as a driving source of the dynamo currents. These studies attempted to deduce the periodicity in the magnetic field variation to identify the mode of the wave responsible for the dynamo currents. Although Parkinson (1982) reported that no signature of the atmospheric quasi 2-day wave was detected in the magnetic field, Ito et al. (1986) found, on the basis of a simulation of the ionospheric currents, that the geomagnetic field variation originated from the wind field probably has sufficient amplitude to be detected on the ground. Takeda and Yamada (1989), using magnetic $H$ variations at low- and middlelatitude observatories, found peaks at 45 and 58 hours in the power spectrum, and discussed their relationship with the quasi 2-day wave in the middle atmosphere. Forbes and Leveroni (1992) and Kohsiek et al. (1995) showed the exis-

Copy right (C) The Society of Geomagnetism and Earth, Planetary and Space Sciences (SGEPSS); The Seismological Society of Japan; The Volcanological Society of Japan; The Geodetic Society of Japan; The Japanese Society for Planetary Sciences. tence of a quasi 16-day oscillation in the geomagnetic field, and concluded that a planetary wave with a period of 16 days drives it.

The results mentioned above were obtained mainly from the analyses of the intensity of $S q$. On the other hand, a variation certainly exists also in the daily profile pattern of $S q$; it is not rare that the pattern of the daily variation fluctuates bewilderingly from day to day. Such a variation means that the equivalent current system of $S q$ varies in its shape as well as in its intensity. Many authors have reported that the vortices of the overhead current of $S q$ in both hemispheres change not only the intensities but also the locations of the foci every day (e.g., Hasegawa, 1960). Although Suzuki (1979) suggested that the latitudes of the foci appear to vary with a period of 7-10 days, it has not yet been confirmed analytically whether the temporal variation of the foci locations is composed of any periodic variations. It is reasonable to suppose, however, that the irregular variation in the morphology of the $S q$ current is a consequence of overlapping contributions from various kinds of driving sources having different time scales. This idea is supported by the existence of, at least, a few periodicities in the intensity variation of $S q$.

In this paper, we have analyzed the day-to-day variation of $S q$ at a low-latitude station, Chichijima (CBI; geographic coordinates $27.15^{\circ} \mathrm{N}, 142.30^{\circ} \mathrm{E}$; geomagnetic $\left.18.11^{\circ} \mathrm{N}\right)$. The object of the analysis is to deduce any periodicity in the day-to-day variation both of the intensity and pattern of daily variation. By using a more sophisticated method of analysis than those used in prior work, three periodic variations in the range $<\sim 10$ days were extracted clearly from 
Table 1. Locations of stations and the intervals of data used in this study. The values are reprinted from World Data Center for Geomagnetism, Kyoto, DATA CATALOGUE (1999).

\begin{tabular}{|c|c|c|c|c|c|c|}
\hline & & & \multicolumn{2}{|c|}{ Geographic } & \multicolumn{2}{|c|}{ Geomagnetic } \\
\hline \multicolumn{2}{|c|}{ Station Name } & Year & Latitude & Longitude & Latitude & Longitude \\
\hline Chichijima & (CBI) & 1970-92 & $27.15^{\circ}$ & $142.30^{\circ}$ & $18.11^{\circ}$ & $211.30^{\circ}$ \\
\hline Memambetsu & (MMB) & 1970-92 & $43.90^{\circ}$ & $144.20^{\circ}$ & $34.93^{\circ}$ & $210.78^{\circ}$ \\
\hline Kakioka & (KAK) & 1970-92 & $36.23^{\circ}$ & $140.18^{\circ}$ & $26.94^{\circ}$ & $208.29^{\circ}$ \\
\hline Guam & (GUA) & $1970-92$ & $13.58^{\circ}$ & $144.87^{\circ}$ & $4.89^{\circ}$ & $215.26^{\circ}$ \\
\hline Port Moresby & (PMG) & 1970-91 & $-9.40^{\circ}$ & $147.15^{\circ}$ & $-17.68^{\circ}$ & $220.20^{\circ}$ \\
\hline
\end{tabular}

the data. Although the variations are characterized primarily by the amplitude oscillation of an $S q$-like daily variation, a fraction of the oscillation indicating the variation of the daily pattern is apparent in the data. Additionally, we also confirmed that the oscillation has some spatial extent across the geomagnetic equator. The results of the analysis give us important clues to ascertain the mechanisms of the variation of the global $S q$ current.

\section{Data Analysis}

The method used in this study is based on principal component analysis (PCA). PCA is widely used in multivariate analyses for reduction of dimensionality (e.g., Krzanowski, 2000), which offers a more concise description of the observed data and helps to extract significant information from the data. Several papers have described the application of the method for $S q$ analysis in the time domain (Vertlib and Wagner, 1970; Golovkov et al., 1978; Rajaram, 1980; Xu, 1989). These papers demonstrated the method's usefulness in extracting predominant patterns from a variety of profiles of the geomagnetic daily variation. The objects of PCA in the prior studies were, however, restricted to 24-hour variation; the question of how a daily variation correlates with the variation on the next or previous day was not a focus of previous work. In this study, the method is applied to successive $n$-day data, as described later, to decompose the $n$-day variation into any systematic day-to-day variations. This modified method enables us to see how the day-to-day variation of $S q$ is composed of different kinds of variation.

We apply the method to the geomagnetic hourly values of the $Z$ component observed at CBI. The station is located in the northern part of Chichijima Island in the northwestern Pacific Ocean (Fig. 1 and Table 1). Figure 2 shows the mean daily variation of geomagnetic northward $(X)$, eastward $(Y)$, and downward $(Z)$ components of CBI averaged over 19701992. Deviations from the nocturnal mean values $(0 \mathrm{~h}-3 \mathrm{~h}$ and $21 \mathrm{~h}-24 \mathrm{~h} \mathrm{LT}$; LT $=\mathrm{UT}+9 \mathrm{~h}$ ) are displayed in this figure. The daily regular variation is apparent in each component and the profile of $Z$, which is used in the present analysis, is characterized by a minimum just before local noon. The maximum deviation from the nighttime level is $-19 \mathrm{nT}$ in this averaged profile.

Figure 3 shows an example of the temporal variation of the three components in March 1990, when magnetic storms repeatedly occurred. The variation of the Dst index in the month is also shown as a reference for global geomagnetic

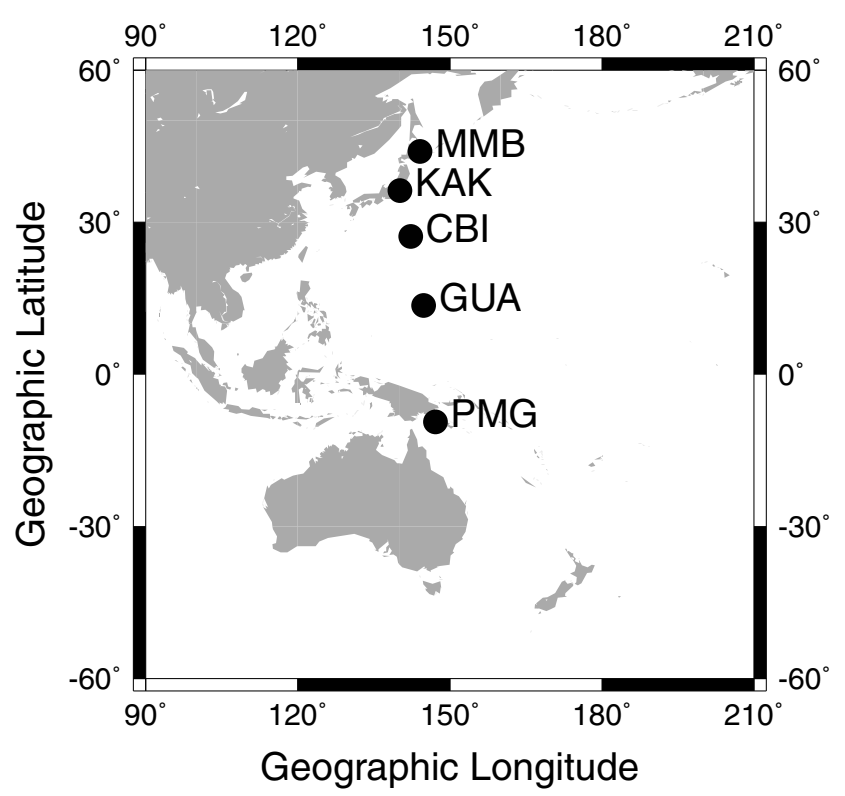

Fig. 1. Location of stations.

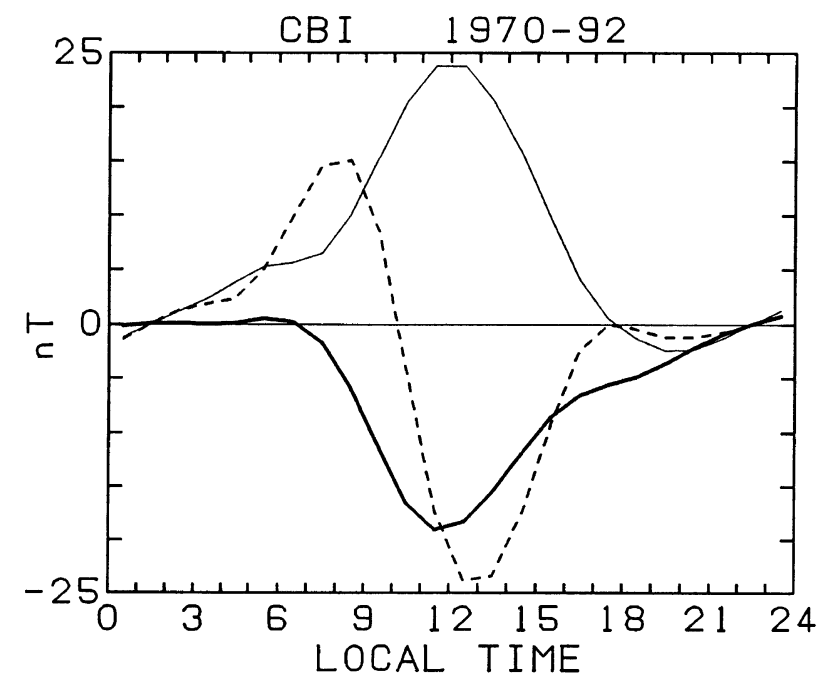

Fig. 2. Mean daily variation of the $X$ (thin line), $Y$ (dashed line), and $Z$ (thick line) components at Chichijima averaged over 1970-1992. Deviations from the nocturnal mean values $(0 \mathrm{~h}-3 \mathrm{~h}$ and $21 \mathrm{~h}-24 \mathrm{~h} \mathrm{LT})$ are displayed. 


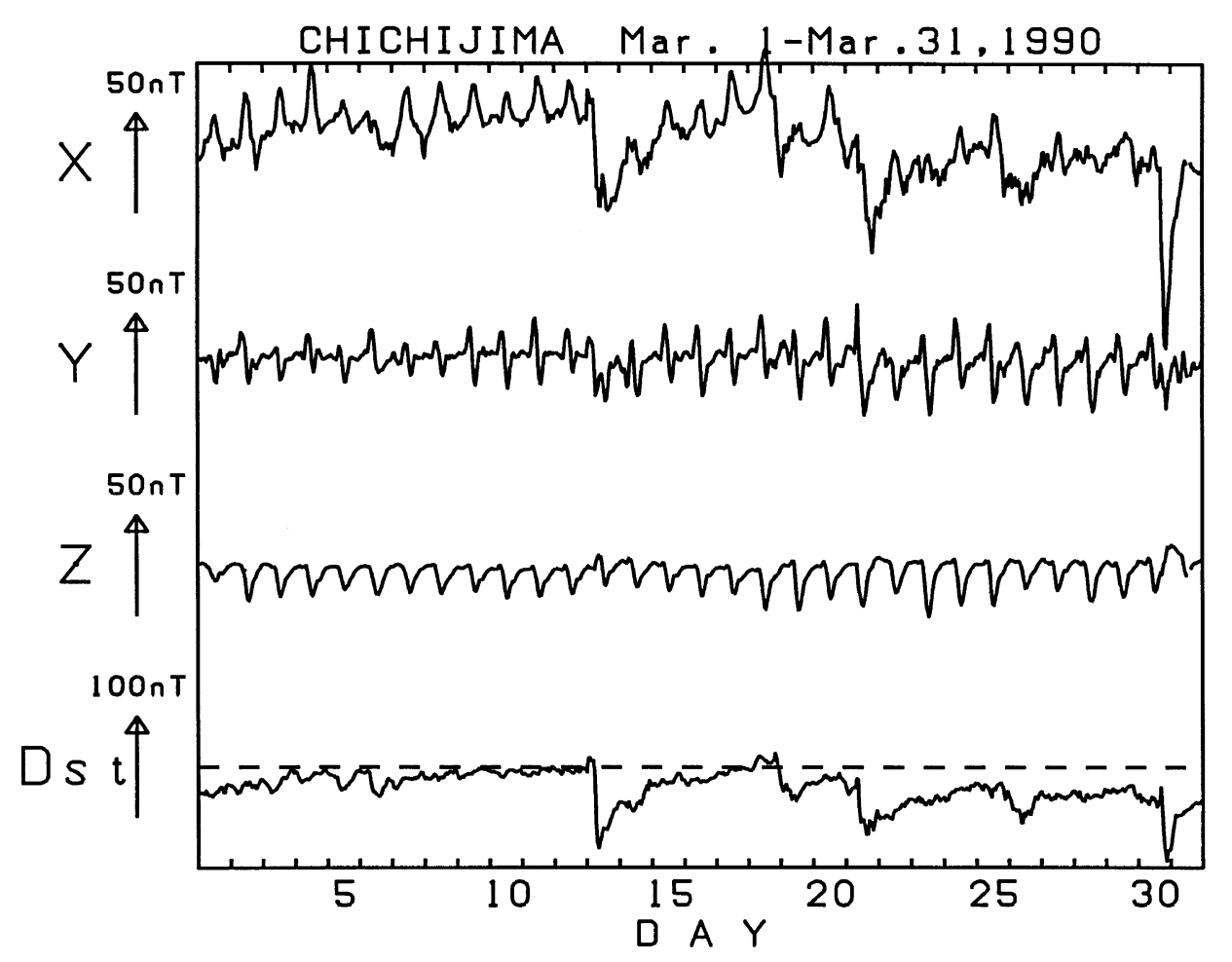

Fig. 3. The geomagnetic variation of the $X, Y$, and $Z$ components at Chichijima and the $D$ st index on March 1-31, 1990. The zero level of the Dst index is indicated by the dashed line.

activity. The index remained negative during the month after a sudden decrease on March 13, which was followed by multiple occurrences of geomagnetic storms on days 18, 21, and 30. While daily regular variations are clear on geomagnetically quiet or moderately disturbed days, the geomagnetic disturbance field contaminates the daily variations in the $X$ and $Y$ components during storm periods. It can be seen, however, that the rapid magnetic variations found in $X$ and $Y$ during the storms are filtered out from the variation in $Z$, and the profile of the $Z$ component maintains the regular daily variation throughout the month even in the intervals of the severe magnetic storms. The attenuation of high-frequency variation in $Z$ suggests that the conductance of the island is extremely high, so that the magnetic field of the vertical component incident from an upper source is effectively shielded by the ground. This idea is unfounded at present because an electromagnetic survey in and around the island has never been carried out.

This unique feature of the $Z$ component at CBI is useful for statistical studies of the daily variation, especially for the day-to-day variation, because more samples of dailyvariation pattern are available for the investigation. The analysis of the day-to-day variation of $S q$ is considerably difficult in general because the quiet period rarely exceeds even a week. We adopt a more moderate criterion of 'quiet day' than is usually used to collect more samples for statistics: if any of eight values of the $K p$ indices during a day are not larger than $5+$, the $Z$ data of the day are used for the analysis. The results of the analysis described in the next section were almost the same even when the criterion of the $K p$ was $4+$ though the number of available samples was decreased to one-third.

If the ionospheric current is the principal origin of the day-to-day variation of the daily pattern, the variation should probably be reduced considerably in the nighttime due to the decrease of the ionospheric conductivities, and should be strongly dependent on the local time. Therefore 24 hourly values in a local day are used as the minimum unit in the analysis. (As shown in the next section, this approach appears valid and the treatment makes the interpretation of the analytical results easier.) First of all, the linear trend during a local day is removed using six hourly values in the nighttime $(0 \mathrm{~h}-3 \mathrm{~h}$ and $21 \mathrm{~h}-24 \mathrm{~h} \mathrm{LT})$. This preparation reduces the effect of long-range variations, which is outside the present focus of the analysis, and increases the precision of the results. The resultant hourly values in a local day denoted by $i$ can be expressed by a 24 -element column vector,

$$
z_{i}=\left(z_{i}(0), z_{i}(1), \ldots, z_{i}(23)\right)^{T},
$$

where $T$ represents the transposed matrix. By using this notation, successive hourly values for $2 M+1$ days centered on the day $i$ can expressed as a $24(2 M+1)$-element column vector as follows:

$$
\boldsymbol{Z}_{i}=\left(\begin{array}{c}
z_{i-M}-\bar{z}_{i} \\
z_{i-M+1}-\bar{z}_{i} \\
\vdots \\
z_{i}-\bar{z}_{i} \\
\vdots \\
z_{i+M-1}-\bar{z}_{i} \\
z_{i+M}-\bar{z}_{i}
\end{array}\right),
$$


where $M$ is the half width of a sample in day, and

$$
\bar{z}_{i}=\frac{1}{K} \sum_{k=-M}^{M} z_{i+k} \quad(K=2 M+1)
$$

is the averaged daily variation over the $K$ days. By subtracting $\bar{z}_{i}$ from the hourly values on each day, $\boldsymbol{Z}_{i}$ represents only a day-to-day variation of $S q$ during a given interval of $K$ days. Then we calculate all the vectors, $\left\{\boldsymbol{Z}_{i}, i=\right.$ $1,2, \ldots, N\}$, from the $Z$ data from 1970 to 1992 , sliding the window by one day under the condition that neither any $K p$ in the period exceeded $5+$ nor missing values are contained.

Our aim is finding some predominant patterns of the dayto-day variation in the variety of observed waveforms. Because each vector $\boldsymbol{Z}_{i}$ represents a $K$-day variation, this work is reduced to a statistical calculation by applying PCA to the population of the vectors. The principal components are obtained by solving an eigenvalue equation,

$$
\boldsymbol{C b}_{i}=\lambda_{k} \boldsymbol{b}_{k} \quad(k=1,2, \ldots, 24 K)
$$

for any $k$, under the following constraint on the eigenvectors for normalization,

$$
\left|\boldsymbol{b}_{k}\right|=1
$$

Here, $\boldsymbol{b}_{k}$ and $\lambda_{k}$ are $k$-th eigenvector and eigenvalue, respectively, of the covariance matrix $(24 K \times 24 K)$,

$$
\boldsymbol{C}=\frac{1}{N} \sum_{i=1}^{N}\left(\boldsymbol{Z}_{i}-\overline{\boldsymbol{Z}}\right)\left(\boldsymbol{Z}_{i}-\overline{\boldsymbol{Z}}\right)^{T},
$$

where $N$ is the number of samples, and

$$
\overline{\boldsymbol{Z}}=\frac{1}{N} \sum_{i=1}^{N} \boldsymbol{Z}_{i}
$$

is the mean vector of the samples. The symmetry of the matrix $\boldsymbol{C}$ leads to the fact that all the eigenvalues are real and nonnegative. Next, the eigenvalues are placed in descending order for convenience (as $\lambda_{1} \geq \lambda_{2} \geq \lambda_{3} \geq \cdots \geq 0$ ). The matrix $\boldsymbol{C}$ was numerically reduced to tridiagonal form by the similarity transformations, and the $Q R$ algorithm was used to calculate the eigenvalues of the matrix (e.g., Press et al., 1992).

The eigenvectors and the eigenvalues thus obtained can be interpreted as follows. Regarding each sample, $\boldsymbol{Z}_{i}$, as a representation of a point in the $24 K$-dimensional phase space, the distribution of the points in the space indicates the variety of the day-to-day variation. The eigenvectors form a normalized orthogonal basis in phase space, and the set of the vectors can be regarded as a new coordinate system in the space different from the coordinate of the original variables. The variance of the distribution of the projection of $\boldsymbol{Z}_{i}-\overline{\boldsymbol{Z}}$ onto the $\boldsymbol{b}_{k}$-axis is equivalent to the corresponding eigenvalue $\lambda_{k}$. The eigenvector $\boldsymbol{b}_{1}$, corresponding to the largest eigenvalue $\lambda_{1}$, represents the axis along which the population of the points is most stretched in the phase space, and similarly, $\boldsymbol{b}_{2}$ is the axis representing the second most variable direction. The points are less scattered along the axes of higher degree eigenvectors. Consequently, the principal components, which are $24 K$ patterns of the dayto-day variation in $K$ days, can be deduced and ordered by their significance. Each pattern of the day-to-day variability is expressed by the direction of the eigenvector in phase space. The eigenvectors with large eigenvalues are predominant variation patterns in $K$ days, and we are mostly interested in the direction of those eigenvectors. Other information is obtained from the individual point in phase space. For a specific eigenvector, the amplitude of the pattern of each $K$-day data, which is the projection of the sample vector onto the axis, can be obtained. The temporal variation of the amplitude is important information for the interpretation of the eigenvector.

In practice, the width of the window, $M$, should be selected according to the time-scale concerned. $M$ is set to 5 (the size of the window is 11 days) in this analysis, which means that the target of the analysis is restricted to those phenomena having time scales shorter than several days. A total of 2409 samples are available using this size window for the $Z$ data of CBI from 1970 to 1992 . If larger size window were used, the hourly values in the window would more frequently contain data to be omitted (either missing data or having a value during the interval of $K p>5+$ ), and only a small number of the vectors would be available.

\section{Results of the Analysis}

Eigenvalues of the leading ten components among 264 $(=24 \times 11)$ principal components are listed in Table 2 . The percentage variance, defined as the ratio of the eigenvalue to the total sum of eigenvalues, is $12.9 \%$ for the first component, and the cumulative percentage variance up to 10 th component amounts to $65 \%$ of the total variance. Each component except for 7 th and 8th is associated with the dayto-day variation having a characteristic period as will be explained later. Four kinds of variations, 2-day, 3-day, 5-6day, and $\sim 15$-day oscillations, are found in the first ten components as is summarized in Table 2 . The oscillation with a period of about 15 days expressed by the 1 st and 2 nd components is beyond the scope of this paper because the profiles of the variation cannot be deduced precisely due to period being longer than the size of the window used in the present analysis. Consequently, we will describe in this section the components related to the remaining three periods, and at the same time, the correspondence of these components to the periodic variations will be explained.

Table 2 shows that, among the leading ten components, successive two components represent the same periodic variation. Those pairs of the components will be discussed first. Next, we will briefly examine some of the subsequent components which also express the same periodicities as those found in the leading ten components. In the last part of this section, a preliminary result of another analysis on the spatial structures of the oscillations will be shown.

\section{5-6-day oscillation}

Figures 4(a) and (b) show the eigenvectors of the 5th and 6 th principal components, $\boldsymbol{b}_{5}$ and $\boldsymbol{b}_{6}$, respectively, presented by the Day-LT contour plots. Though each eigenvector represents a pattern of 11-day time series, such a twodimensional presentation is useful to clarify the oscillation 
Table 2. (a) Eigenvalue, (b) percentage variance, (c) cumulative percentage variance, and (d) estimated period (in days) of the first ten components. Symbol " - " means that the eigenvector of the component does not represent any periodic variation. See text as to the method of estimation of the periods.

\begin{tabular}{cccrrrrrrrr}
\hline Component & 1 & \multicolumn{1}{c}{2} & \multicolumn{1}{c}{3} & \multicolumn{1}{c}{4} & \multicolumn{1}{c}{5} & \multicolumn{1}{c}{6} & \multicolumn{1}{c}{7} & \multicolumn{1}{c}{8} & 9 & 10 \\
\hline (a) & 359.1 & 246.1 & 172.9 & 164.4 & 157.6 & 156.0 & 147.5 & 145.1 & 140.6 & 136.4 \\
(b) & 12.9 & 8.8 & 6.2 & 5.9 & 5.6 & 5.6 & 5.3 & 5.2 & 5.0 & 4.9 \\
(c) & 12.9 & 21.7 & 27.9 & 33.8 & 39.4 & 45.0 & 50.3 & 55.5 & 60.5 & 65.4 \\
(d) & $\sim 15$ & $\sim 15$ & 2 & 2 & $5-6$ & $5-6$ & - & - & 3 & 3 \\
\hline
\end{tabular}

structure fixed with LT. In this figure, the profile on each Day is characterized by a single maximum or minimum just before local noon, and the vectors express the variation pattern in which the data in the daytime is larger in some days and smaller in other days than the 11-day mean level.

The eigenvector profile for each Day is similar to the mean daily variation in the $Z$ component (Fig. 2) except for the sign of the midday peak. This means that the eigenvectors primarily represent the day-to-day variation of the amplitude of the daily variation without evident fluctuation of the daily pattern. The positive and negative anomalies in the daytime continue for two or three days and alternate almost regularly, which reveals the existence of a 5- or 6-day oscillation in the day-to-day variation confined to the sunlit hours.

On the other hand, the day-to-day variation is inactive in the nighttime (0-6h, 16-24h). Since the hourly values deviating from the nocturnal $(0-3 \mathrm{~h}, 21-24 \mathrm{~h})$ mean are used for each day in this analysis, it is reasonable that the nighttime values of the eigenvectors deduced here is smaller than those in the daytime. However the facts that the value is steady at around the zero level during the interval and the fluctuation is sharply confined within $6 \mathrm{~h}-16 \mathrm{~h}$ cannot be attributed to the data processing. This suggests that the oscillation is reflected upon by the solar radiation, and that the ionospheric conductivity affects the day-to-day variation of this mode.

Next, we calculate a score for each component, using the following scalar product of a sample vector with an eigenvector:

$$
a_{i k}=\left(\boldsymbol{Z}_{i}-\overline{\boldsymbol{Z}}\right)^{T} \boldsymbol{b}_{k}
$$

where $a_{i k}$ is the score of $i$-th day for $k$-th component. While the eigenvector represents a pattern of 11-day magnetic variations, the score gives the amplitude of the pattern on a given 11-day interval. The auto-correlation coefficients derived from the time series of the scores,

$$
R_{k}(\tau)=\frac{f_{k}(\tau)}{f_{k}(0)}
$$

for 5th and 6th components are displayed in Fig. 4(c). Here,

$$
f_{k}(\tau)=\frac{1}{N_{\tau}} \sum_{i} a_{i k} a_{i+\tau, k}
$$

is the auto-correlation function with a time lag of $\tau$ days for $k$-th component. The summation is calculated for every existing pair of $a_{i k}$ and ${ }_{i+\tau, k} ; N_{\tau}$ is the number of the pairs.
The positive peaks of the correlation are at time lags of 56 days, while the negative peaks appear at 3 days, which supports the existence of the periodicity of 5-6 days in both components. (In practice, the correlations at lags of 5 or 6 days are not high enough to be statistically significant, and the recurrence is ambiguous. This is probably due to the amplitude variability of the scores, as explained below, and also to the distorted waveform as seen in Figs. 4(a) and (b).) Figure 4(d) is an example of time series of the daily scores of the 5th and 6th components overlaid (thin and dashed lines, respectively) during two months, May-June 1976, where the value on a day was calculated from hourly values within 5 days before and after the day. The figure shows that the 56-day oscillation continuously exists in each profile though the amplitude is modulated.

Although these eigenvectors, $\boldsymbol{b}_{5}$ and $\boldsymbol{b}_{6}$, are orthogonal to each other in the 264-dimensional phase space and, consequently, the scores for both components have no correlation at all, the configurations of the two eigenvectors are apparently in a particular relationship. In Figs. 4(a) and (b), $\boldsymbol{b}_{5}$ has positive values on Days -3 to -1 in the daytime and is negative on Days 0 to +2 , while $\boldsymbol{b}_{6}$ is positive on Days -4 to -2 and +2 to +3 , and negative on Days -1 to +1 . In short, the alternation from positive to negative (negative to positive) values of $\boldsymbol{b}_{5}$ appears to occur during the interval the negative (positive) anomaly of $\boldsymbol{b}_{6}$. Both patterns represent the same oscillation but show a 'phase-quadrature' relationship. This feature is clearly demonstrated in Fig. 4(d): the profiles of the two oscillations are similar but the variation of 5 th component constantly leads that of 6th component by 1-2 days. Moreover, the envelopes of $a_{5}$ and $a_{6}$ show almost the same temporal variation along with the regular oscillations though the amplitudes of the oscillations vary with time. This is consistent with the fact that $\lambda_{5}$ and $\lambda_{6}$ are almost the same $\left(\lambda_{5} / \lambda_{6}=1.01\right)$ because the eigenvalue gives the variance of the scores for the component. The two vectors that are orthogonal but quadrature to each other, therefore, express the same phenomenon of the 5-6-day oscillation. This is analogous to the fact that every sinusoid with an arbitrary phase can be expressed by a linear combination of a sine function and a cosine function.

The following is likely to be the reason why an oscillation is expressed by two patterns. When 11-day intervals are selected as the samples for PCA from a time series including the oscillation, the phase of the oscillation relative to the window is different from sample to sample. Even if the oscillation is stationary throughout the time series, the phase of the oscillation relative to the window is not constant for 
(a)

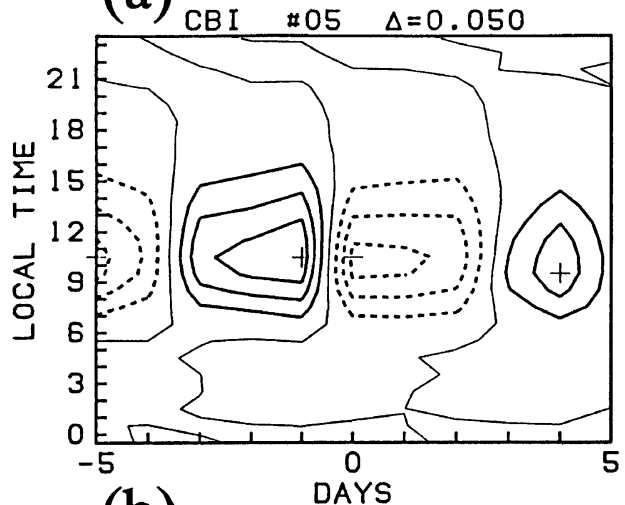

(b)

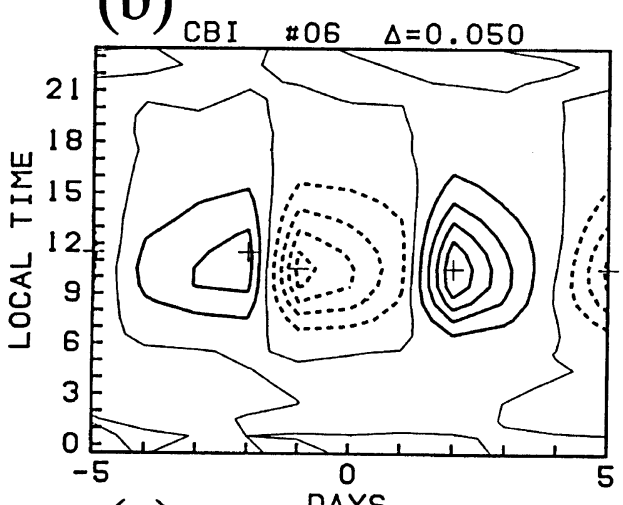

(c)

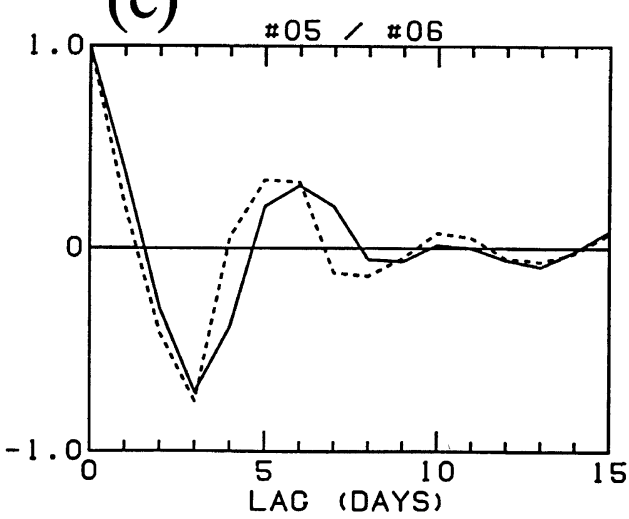

(d) (\#05, \#06) 1976

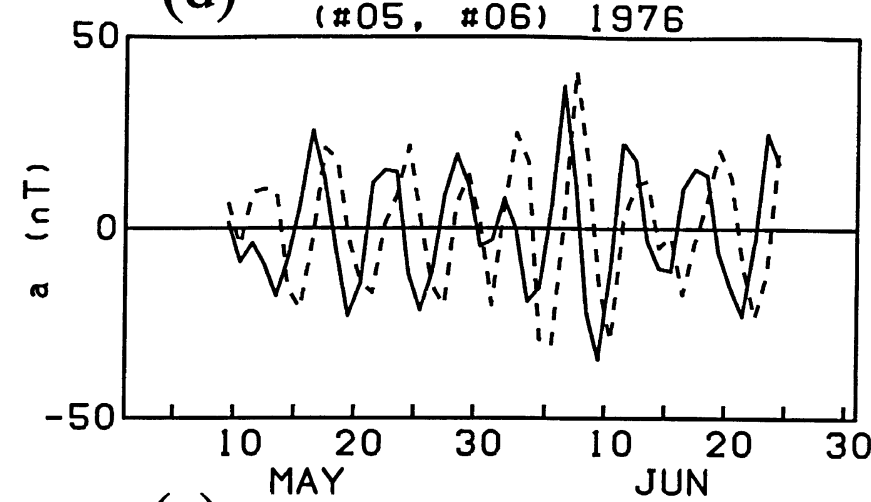

(e)

(\#05. \#06) 1976

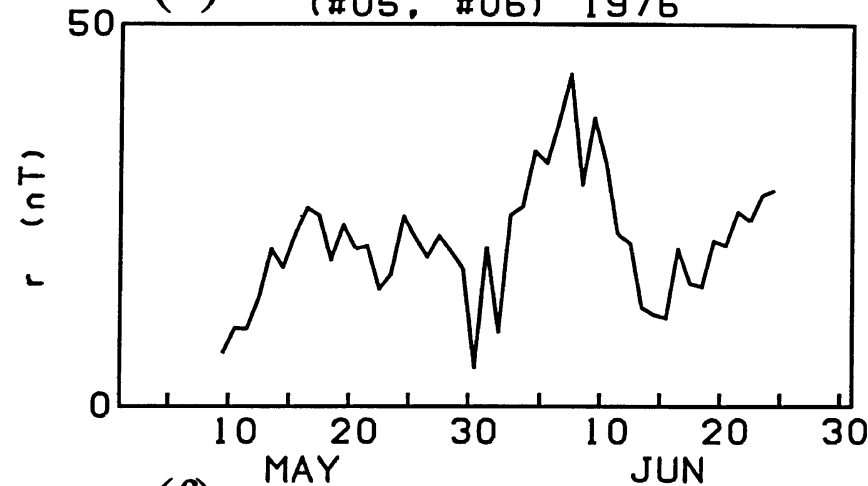

(f)

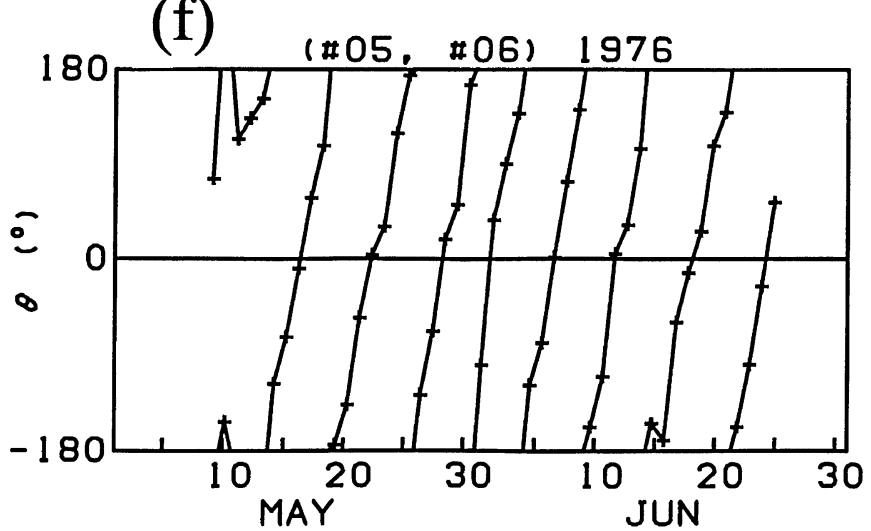

Fig. 4. (a), (b) Eigenvectors of the 5th and 6th principal components, respectively. Contours are drawn at every 0.05 . Thick and dashed contour lines represent the positive and negative values, respectively. (c) The auto-correlation coefficients of the daily scores of the 5th and 6th principal components (thin and dashed line, respectively). (d) The daily scores of the 5th and 6th principal components (thin and dashed line, respectively) in May and June 1976. (e) The daily amplitude of the 5-6-day oscillation. (f) The daily phase angle of the 5-6-day oscillation.

every sample. Therefore, when all the samples are treated in a statistical analysis, the oscillation cannot be expressed by one component but inevitably by two components that apparently differ only in phase by $90^{\circ}$ within the window.

We define the following variables based on the analogy with the sinusoid:

$$
\begin{aligned}
& r=\sqrt{a_{5}^{2}+a_{6}^{2}} \\
& \theta=\arctan \frac{a_{6}}{a_{5}}
\end{aligned}
$$

where $r$ and $\theta$ represent the amplitude and the phase of the oscillation, respectively (the subscript $i$ is omitted for simplicity). As an example, these two variables calculated from the scores in Fig. 4(d) are shown in Figs. 4(e) and (f), respec- tively. In the figure, the amplitude, $r$, varies with the different time scale from that of the oscillation: after a moderate peak in the middle of May, the amplitude takes the highest value in the first part of June. Considering the fact that the standard deviations (SD) of both scores are $12.5\left(=\sqrt{\lambda_{5}}\right.$ and $\sqrt{\lambda_{6}}$ ), it is notable that the scores reach about $3 \mathrm{SD}$ at the highest peak. In spite of the amplitude variability, the phase angle increases almost constantly during the interval and makes one rotation in 5-6 days even when the amplitude declined. This suggests that the 5-6-day oscillation is present almost continuously though the amplitude is highly variable. To find the correct 'phase speed' of the oscillation, phase differences between successive two days are compiled for all the scores. Figure 5(a) shows the histogram of occur- 
(a)

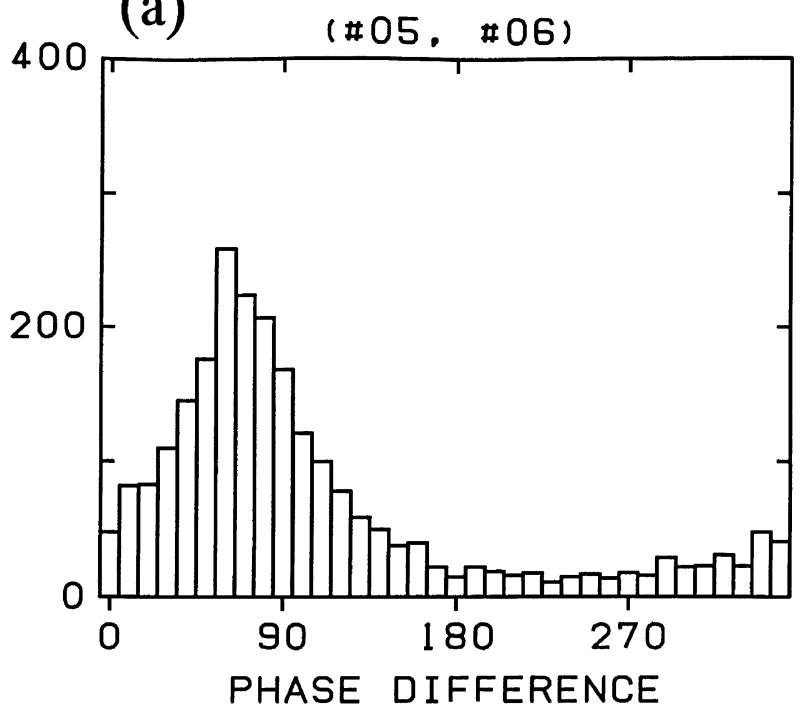

(b)

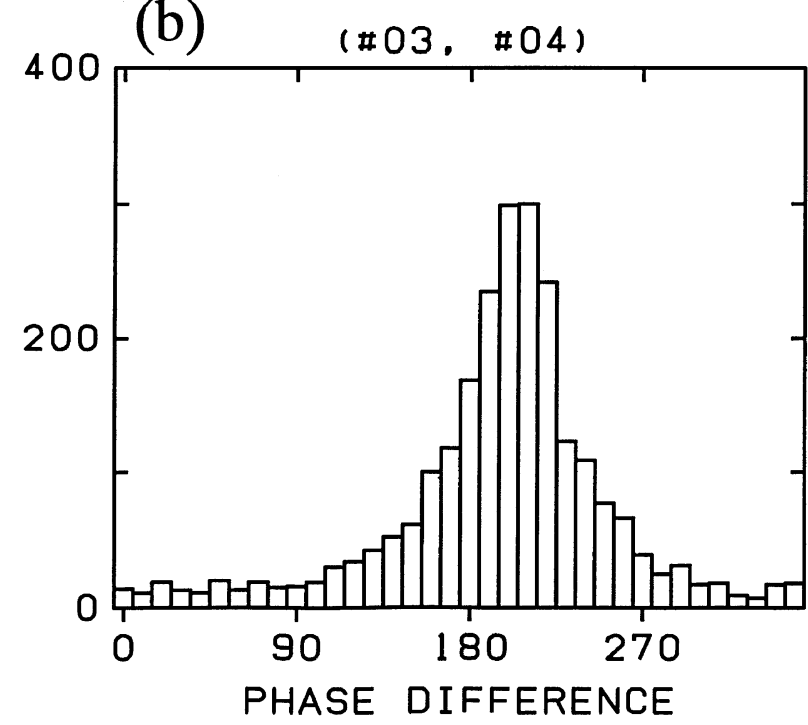

Fig. 5. Histogram of the occurrence frequency of phase differences between successive two days for (a) the 5-6-day oscillation and (b) the 2-day oscillation.

rence frequency of the phase difference. A peak exists at $60^{\circ}-80^{\circ}$, which is equivalent to the period of 4.5-6 days.

Figure 6(a) is a demonstration of how the daily pattern of anomaly changes as the phase proceeds constantly day by day. Here we calculate linear combinations of the two eigenvectors for every $60^{\circ}$ phase angle. Because the variances of the scores of 5 th and 6th components are $\lambda_{5}$ and $\lambda_{6}, \sqrt{\lambda_{5}}$ and $\sqrt{\lambda_{6}}$ are used as typical values of the respective amplitudes. A one-day profile on Day 0 is selected from the vector,

$$
\boldsymbol{Z}=\sqrt{\lambda_{5}} \boldsymbol{b}_{5} \cos \theta+\sqrt{\lambda_{6}} \boldsymbol{b}_{6} \sin \theta
$$

for each phase angle, and these profiles are arranged in order of increasing phase angle. This figure is consistent with the idea that the 5 th and 6th components together represent the 5-6-day oscillation.

Figure 6(a) also shows that the amplitude of the oscillation amounts to $2.8 \mathrm{nT}\left(\theta=240^{\circ}\right)$ and the range during the 6-day profile reaches $5.5 \mathrm{nT}$, which are $15 \%$ and $29 \%$ of the range of the mean daily variation in the $Z$ component at CBI (Fig. 2), respectively. It is noteworthy that the amplitudes of the components were set to respective SDs in the calculation. The range of the oscillation demonstrated in Fig. 6(a) reaches higher value when the scores are larger.

\section{3-day oscillation}

Figures 7(a) and (b) show the eigenvectors of the 9th and 10th principal components, $\boldsymbol{b}_{9}$ and $\boldsymbol{b}_{10}$, respectively. Like those of the 5-6-day oscillation, a peak on each Day appears slightly before noon. The daily profile resembles the mean daily variation of the $Z$ component shown in Fig. 2 while the nocturnal values are almost zero throughout the window. This means that the variation shown in the vectors is probably originated from ionospheric currents. Positive and negative anomalies in the daytime in each pattern continues for 1-2 days and almost regularly alternate, which suggests the predominance of an oscillation with a period of about 3 days. The alternation in the daytime of $\boldsymbol{b}_{9}$ and $\boldsymbol{b}_{10}$ are $90^{\circ}$ out of phase to each other, similar to the relationship between $\boldsymbol{b}_{5}$ and $\boldsymbol{b}_{6}$ (Figs. 4(a) and (b)). Moreover, the variances of these two components, $\lambda_{9}$ and $\lambda_{10}$, are almost the same $\left(\lambda_{9} / \lambda_{10}=1.03\right)$. Therefore it seems reasonable to suppose that the two vectors, which are orthogonal but quadrature to each other, express the same 3-day oscillation.

The day-to-day variation of the daily scores of the 9 th and 10th components, $a_{9}$ and $a_{10}$, are calculated to confirm the existence of the oscillation. Using the scores, the phase and amplitude can be defined in a manner similar to that used in Eqs. (11) and (12). Figure 7(d) gives an example of the temporal variations of the scores in two months. Figures 7(e) and (f) show the estimated phase and the amplitude of the oscillation in the same interval, respectively. Note that the phase proceeds constantly taking about 3 days for one rotation except for the period during which the amplitude is at its lowest level. Figure 7(c) displays the auto-correlation coefficients of the scores derived from all the data used in this analysis. Positive peaks at lags of 3 and 6 days are clear for both components, which is evidence of the presence of the 3 -day oscillation in the profiles of these components.

As in the same manner of Fig. 6(a), using $\sqrt{\lambda_{9}}$ and $\sqrt{\lambda_{10}}$ as typical values of the respective amplitudes of 9 th and 10th components, a time series of successive three days is reproduced from linear combinations of the two eigenvectors for every $120^{\circ}$ phase angle (not shown). The result shows that the largest daily range among the three days is $2.2 \mathrm{nT}$, which amounts to $12 \%$ of the range of the mean daily variation in the $Z$ component.

\section{2-day oscillation}

The eigenvectors of the 3rd and 4th principal components, $\boldsymbol{b}_{3}$ and $\boldsymbol{b}_{4}$, are shown in Figs. 8(a) and (b), respectively. The variation in each Day is predominant only in the daytime while the nocturnal profile is almost flat. This suggests that the variation associated with the eigenvectors is originated from ionospheric currents. There is a midday maximum or minimum every Day, and the daily profile resembles the mean profile of $Z$ component (Fig. 2) though the daily variation described in the eigenvectors frequently changes its sign: the Day having a positive peak is followed by a Day with a negative peak, and vice versa. The eigen- 
(a)

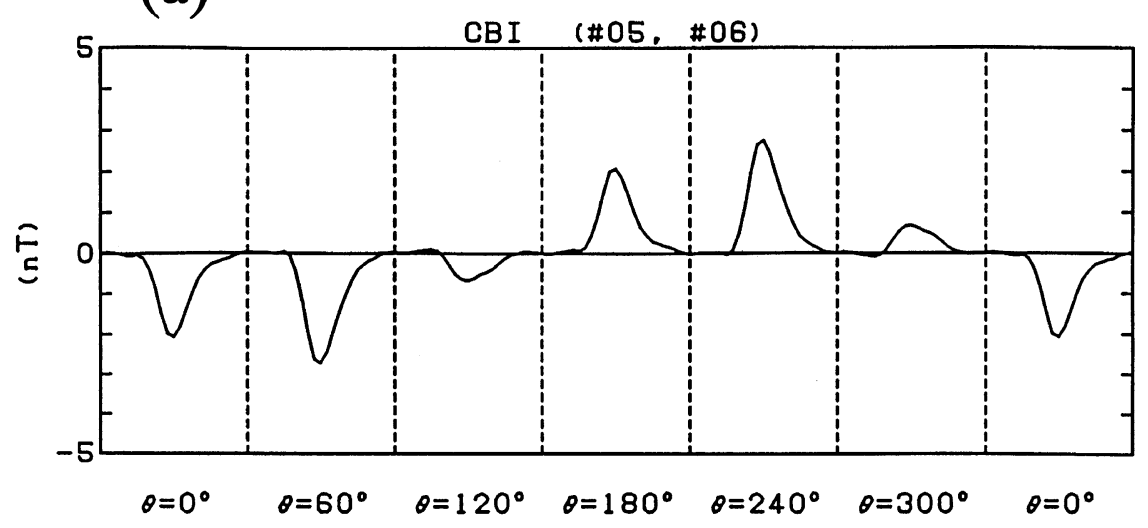

(b)

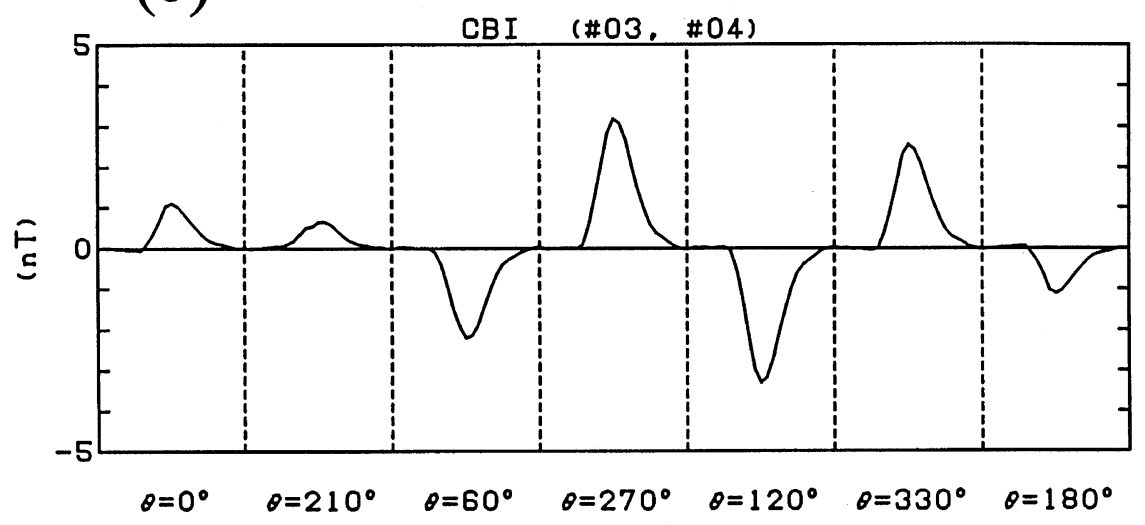

(c)

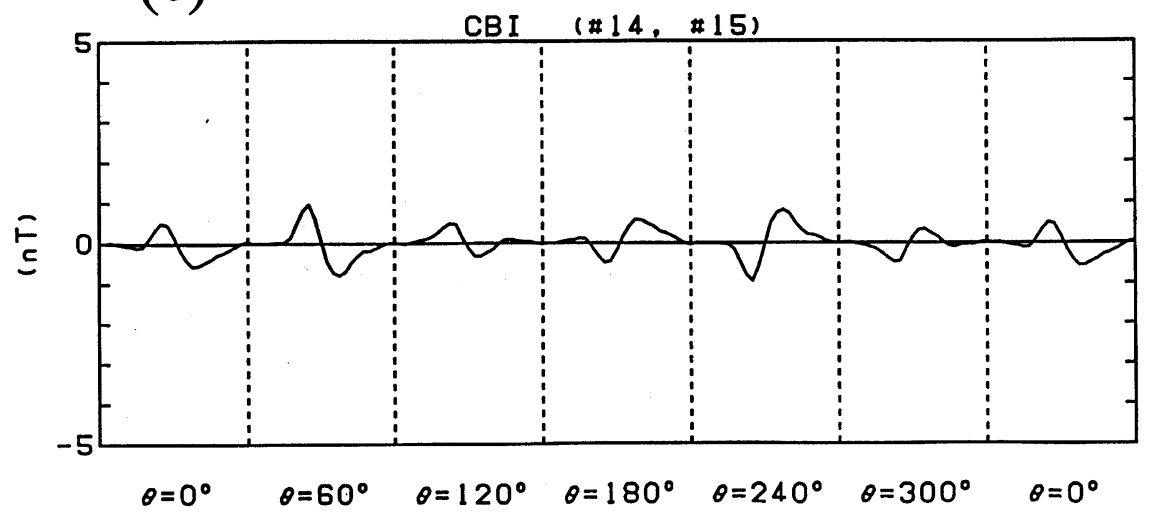

Fig. 6. (a) A demonstration of the 5-6-day oscillation. The eigenvectors, $\boldsymbol{b}_{5}$ and $\boldsymbol{b}_{6}$, are linearly combined for every $60^{\circ}$ phase angle. The amplitudes of the components are assumed to be $\sqrt{\lambda_{5}}$ and $\sqrt{\lambda_{6}}$, respectively. (b) A demonstration of the 2-day oscillation. The eigenvectors, $\boldsymbol{b}_{3}$ and $\boldsymbol{b}_{4}$, are linearly combined for every $210^{\circ}$ phase angle. The amplitudes of the components are assumed to be $\sqrt{\lambda_{3}}$ and $\sqrt{\lambda_{4}}$, respectively. (c) Same as (a), but for the 14 th and 15 th principal components.

vectors, therefore, express the amplitude fluctuations of the daily variation including a 2-day oscillation. Another feature evident from these figures is that the amplitude of the 2-day oscillation is variable in the 11-Day interval. The eigenvector $\boldsymbol{b}_{3}$ shows a pattern with large amplitude of the oscillation in the first and last part of the window, and $\boldsymbol{b}_{4}$ in the middle part of the window.

Figure 8(d) shows the day-to-day variation of the scores of the 3rd and 4th components in June 1971. The sense of the score repeatedly changes from day to day, which manifests the 2-day period oscillation. The 2-day periodicity is confirmed also in Fig. 8(c), where the auto-correlation coefficients derived from all the scores are displayed. The positive peaks are at lags of 2 days and negative at a day, and the coefficients alternately rise and drop in one-day intervals.

In the cases of the 5-6-day and 3-day oscillations mentioned above, each oscillation is expressed by two orthog- 
(a)

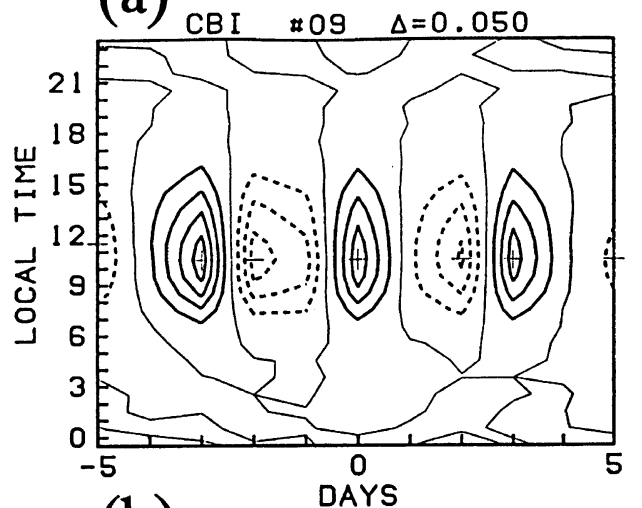

(b)

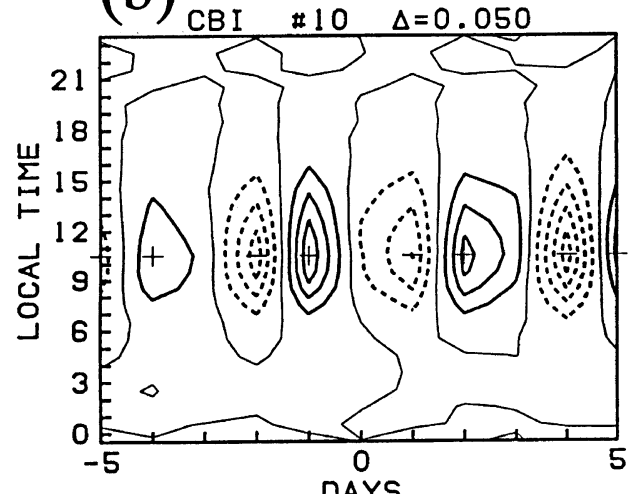

(c)

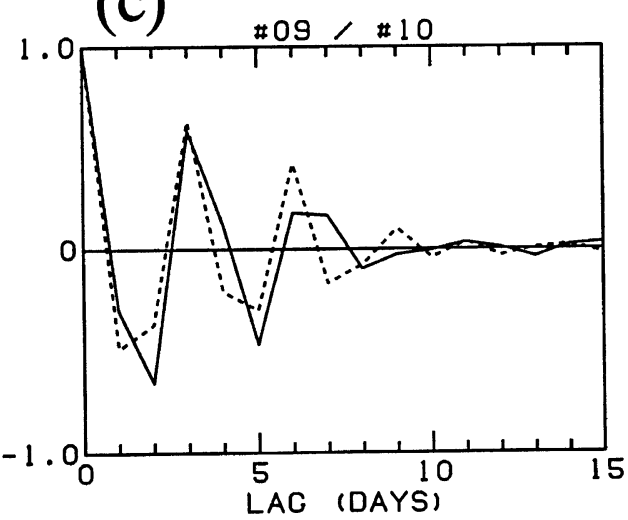

(d)

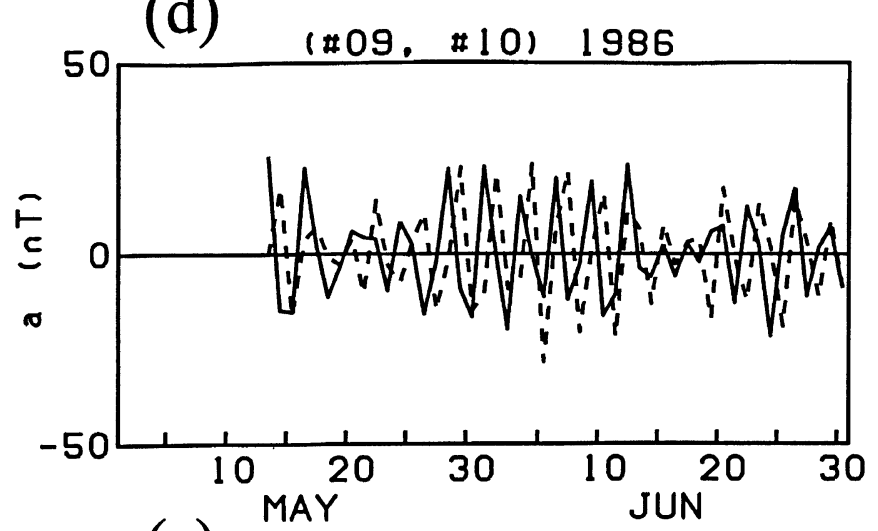

(e)

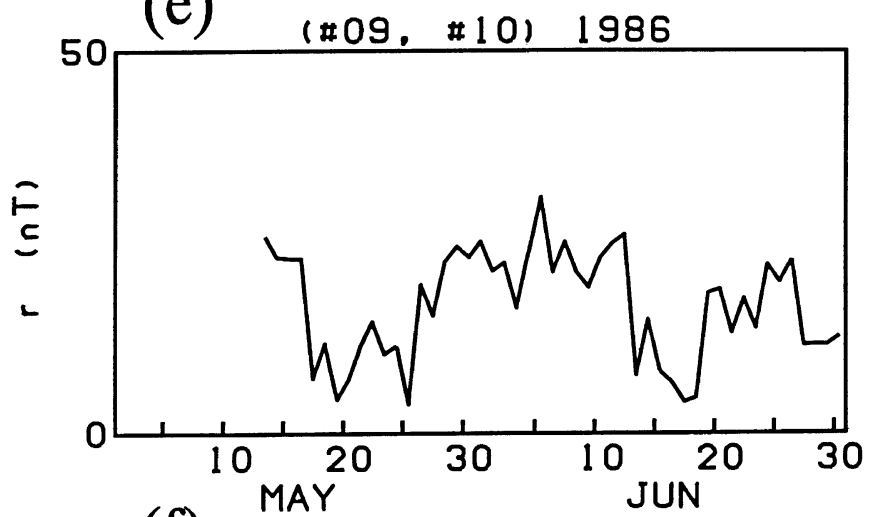

(f)

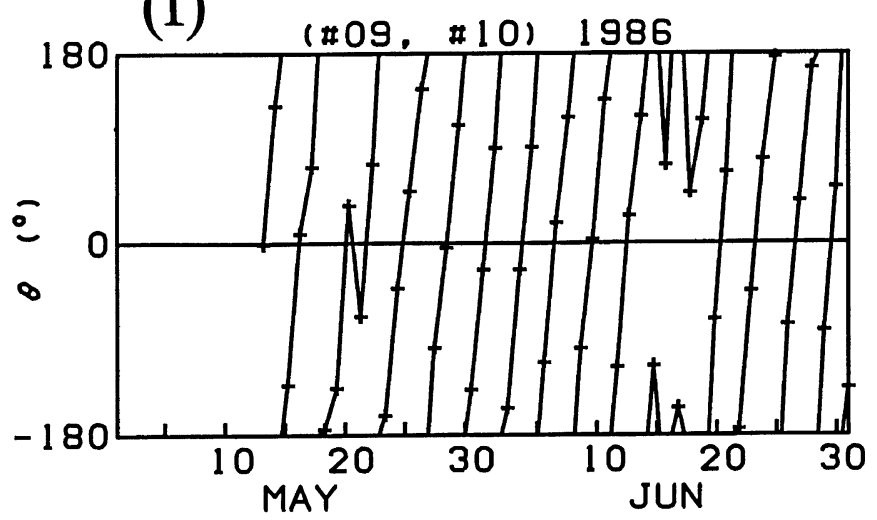

Fig. 7. (a), (b) Eigenvectors of the 9th and 10th principal components, respectively. Contours are drawn at every 0.05 . Thick and dashed contour lines represent the positive and negative values, respectively. (c) The auto-correlation coefficients of the daily scores of the 9th and 10th principal components (thin and dashed line, respectively). (d) The daily scores of the 9th and 10th principal components (thin and dashed line, respectively) in May and June 1986. (e) The daily amplitude of the 3-day oscillation. (f) The daily phase angle of the 3-day oscillation.

onal eigenvectors whose phases in the window are $90^{\circ}$ out of phase. Only one component is, however, sufficient to describe a 2-day oscillation if the daily variation would precisely reverse its pattern everyday. As shown in Figs. 8(a) and (b), this is not the case: the oscillation in $\boldsymbol{b}_{3}$ is activated on Days -3 to -2 and on Days +4 to +5 when it is almost diminished in $\boldsymbol{b}_{4}$, while the disappearance on Day +1 in $\boldsymbol{b}_{3}$ is in the midst of the interval of magnification in $\boldsymbol{b}_{4}$. This indicates that, in $\boldsymbol{b}_{3}$ and $\boldsymbol{b}_{4}$, the amplitude fluctuation of the 2-day oscillation is out of phase to each other, and that the interval of the activation is $7-8$ days. Both components probably express the presence not only of the 2-day oscillation but also of the periodic amplitude fluctuation of the oscillation.
This inference is supported by Figs. 5(b) and 6(b): Figure 5(b) shows the phase difference between successive two days, which shows a peak at $200^{\circ}-210^{\circ}$. The phase differences of $200^{\circ}$ and $210^{\circ}$ indicate the recurrence of cycles of 9 days and 12 days, respectively. Figure 6(b) is a demonstration of the modulation of the oscillation. The linear combinations of the two vectors are calculated for the case that the phase angle proceeds constantly by $210^{\circ}$ day, and daily profiles on Day 0 are arranged in order of the angle through a half cycle. In this reduction, the amplitudes of the oscillations of the two eigenvectors are assumed to be $\sqrt{\lambda_{3}}$ and $\sqrt{\lambda_{4}}$, respectively, representing a typical level of activity. In this case, the maximum amplitude of the fluctuation in the midday amounts to $3.3 \mathrm{nT}\left(\theta=120^{\circ}\right)$, which is $17 \%$ of the 
(a)

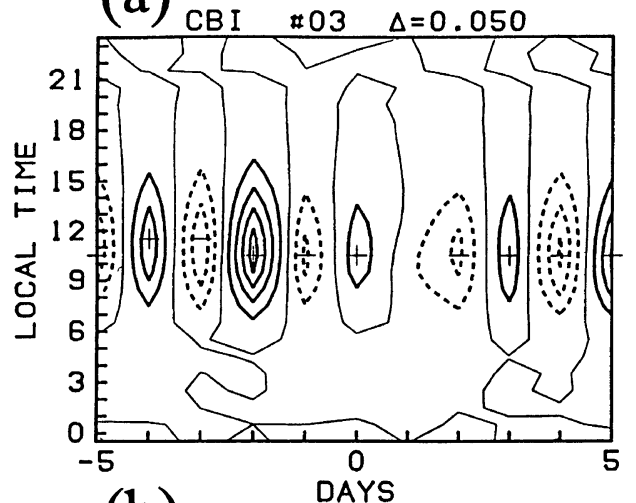

(b) ${ }_{\mathrm{CBI}} \quad$ DAYS $04 \quad \Delta=0.050$

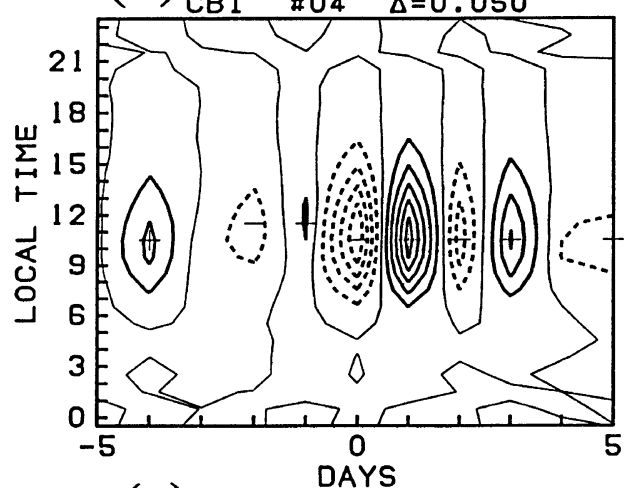

(c)

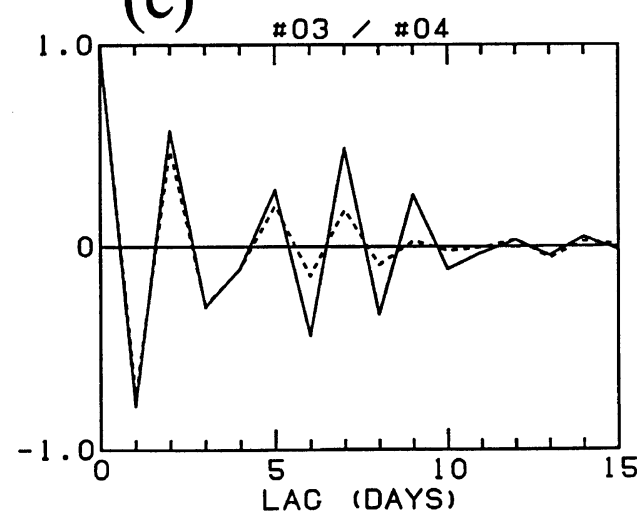

(d) (\#03, \#04) 1971

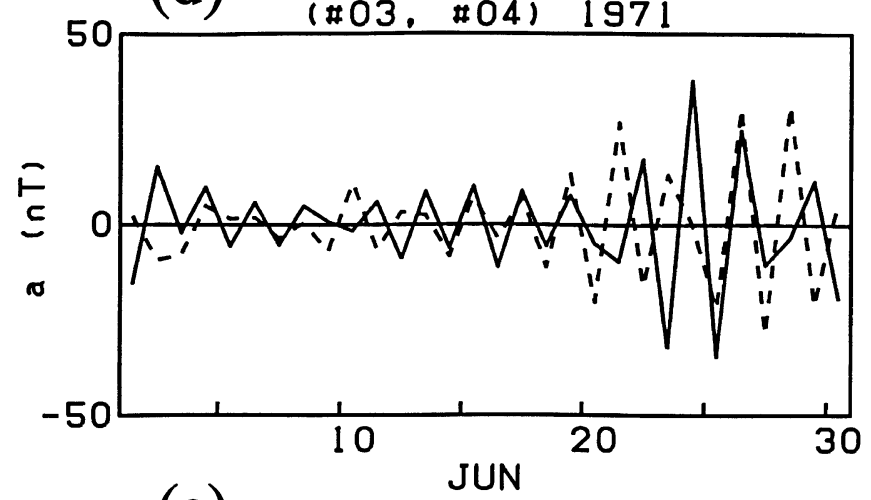

(e)

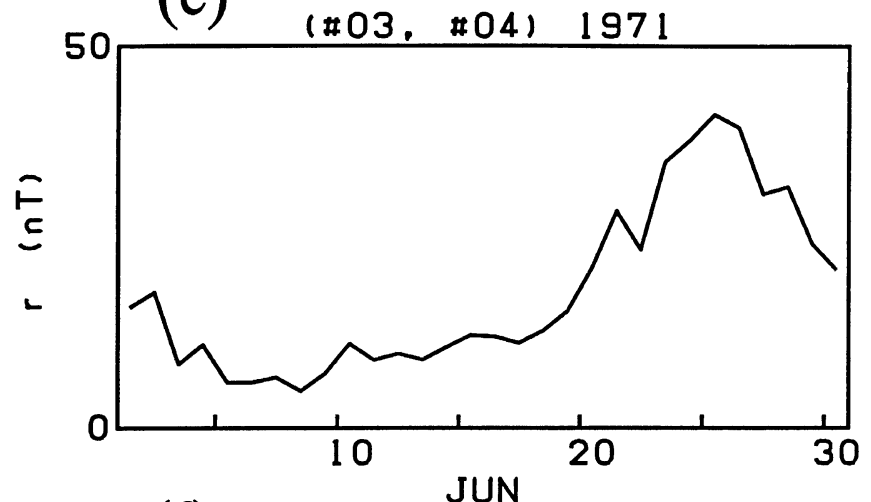

(f)

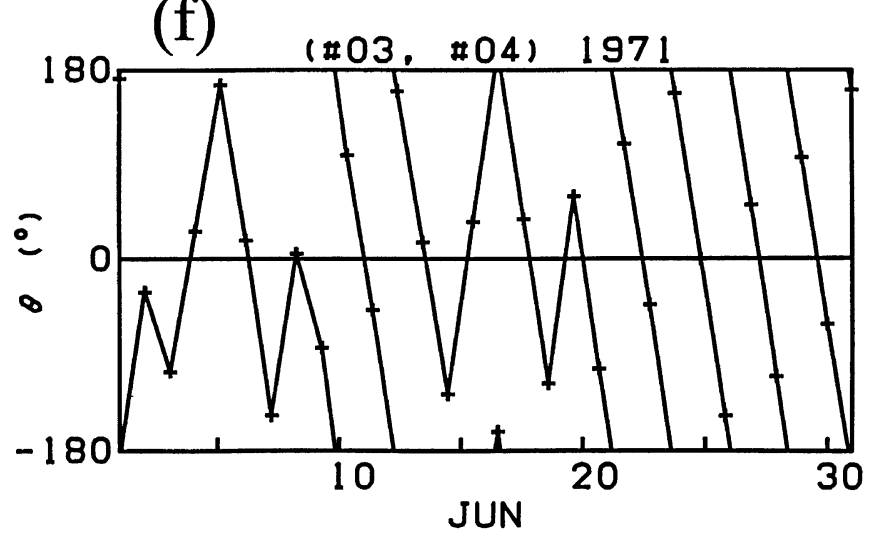

Fig. 8. (a), (b) Eigenvectors of the 3rd and 4th principal components, respectively. Contours are drawn at every 0.05 . Thick and dashed contour lines represent the positive and negative values, respectively. (c) The auto-correlation coefficients of the daily scores of the 3rd and 4th principal components (thin and dashed line, respectively). (d) The daily scores of the 3rd and 4th principal components (thin and dashed line, respectively) in June 1971. (e) The daily amplitude of the 2-day oscillation. (f) The daily phase angle of the 2-day oscillation.

amplitude of the mean daily variation (Fig. 2). This time series shows the 2-day oscillation of the daily variation accompanies an amplitude modulation. Another point to be noticed is the modulation includes an 'interruption' of the oscillation: the alternation of the daily profile is diminished and the reversal of the profile disappears between successive two days of $\theta=0^{\circ}$ and $210^{\circ}$ (and also between $180^{\circ}$ and $30^{\circ}$ in the second half cycle). This feature suggests that the driving force of the ionospheric current responsible for the oscillation has a period slightly different from 48 hours, and a modulation of the current by the daily variation of the ionospheric conductivity is revealed in the oscillation.

\section{Pattern fluctuation of $S q$}

So far, only the eigenvectors with large eigenvalues have been discussed. Each of the principal components interpreted above mainly expresses the fluctuation of the daily amplitude of $S q$. The eigenvectors of the other types of fluctuation are found in the subsequent components. The 14th and 15th eigenvectors are shown in Fig. 9(a) as an example. On each Day, the daily variation of both components has a pair of maxima, positive in the prenoon hours and negative in the postnoon hours or vice versa. The day-to-day alternations of the maxima are clearly regular with a period of 5-6 days, and the phases of the oscillation are apparently different from each other by about $90^{\circ}$. The relation between the two eigenvectors is the same as that of the 5th and 6th components (Figs. 4(a) and (b)) except for the existence of the pair of maxima in a day. Moreover, the phase $\theta$, calcu- 
lated from the scores of both components, steadily increases by about $60^{\circ}$ a day (not shown). These characteristics are similar to the oscillation expressed by 5 th and 6th components. While the 5th and 6th components express the amplitude variation with a period of 5-6 days, the 14th and 15th components represent 'the secondary 5-6-day oscillation', which can also change the daily pattern of $S q$. If this kind of day-to-day variation overlaps with the regular $S q$ variation, the local time of its single maximum in the daytime probably varies earlier or later with 5-6-day period.

The magnitude of the secondary oscillation was evaluated in the same manner as that of Fig. 6(a). Figure 6(c) shows the linear combinations of the two eigenvectors calculated for increments of $60^{\circ}$ in phase angle by using $\sqrt{\lambda_{14}}$ and $\sqrt{\lambda_{15}}$ for respective amplitudes. Maximum daily range among the six days is about $1.7 \mathrm{nT}\left(\theta=60^{\circ}\right)$, which amounts to about $9 \%$ of the range of the mean daily variation.

The secondary 3-day and 2-day oscillations are also found in higher components. Figure 9(b) shows the eigenvectors of the 16 th and 17 th principal components. Both components represent the 2-day oscillation and the relation between the two eigenvectors is the same as that of $3 \mathrm{rd}$ and 4 th components except for the presence of a pair of maxima during a day. Figure 9(c) shows the eigenvectors of the 21 st and 22nd components, which express the secondary 3-day oscillation. By using typical amplitudes of these components, the oscillations were simulated as in Fig. 6(c). Maximum daily ranges of the secondary 2-day and 3-day oscillations were estimated as $2.9 \mathrm{nT}$ and $2.4 \mathrm{nT}$, which are $15 \%$ and $13 \%$ of the range of the mean daily variation, respectively.

The amplitudes of the secondary oscillations of the three periods are smaller than those of the $S q$ itself and the respective primary oscillations. However, they probably play important roles in the pattern fluctuations of $S q$. Remembering that the averaged pattern of the $Z$ component at CBI has a negative maximum just before noon, each oscillation with the pair of maxima superposed on the basic profile regularly moves the midday maximum toward earlier or later hours. This suggests that the east-west movement of the $S q$ focus can be resolved into those periodicities though further investigation on the spatial distributions of the oscillations is needed for the conclusion.

\section{Latitudinal Profiles of the oscillations}

We have obtained the waveforms of oscillations with the three periods in the $Z$ component at CBI. The author is now planning another analysis, as the next step, to investigate spatial structures of the oscillations by using the data of other stations. Here a preliminary result of the analysis will be shown, which clarifies that the oscillations are not local phenomena at $\mathrm{CBI}$.

We have defined $\theta$ of the 5-6-day oscillation by Eq. (12), which gives the phase angle of the oscillation in the $Z$ component at $\mathrm{CBI}$ on each day. $\theta$ can be used as a key parameter in studying whether magnetic field variations at other stations are correlated with the oscillation at CBI. If a daily variation at other location is dependent on $\theta$, it means that it would oscillate synchronously with the $Z$ component of CBI. The location of stations used in this study is shown in Fig. 1 and Table 1. Note that all the five stations are along almost the same meridian.

As can be seen in Fig. 6(a), the midday anomaly of the oscillation changes its sense as $\theta$ proceeds. The daytime variation is positive on the days for which the phase is $150^{\circ}<$ $\theta<270^{\circ}$, and negative on the days when $0^{\circ}<\theta<90^{\circ}$ or $330^{\circ}<\theta<360^{\circ}$. Here all the days used for PCA are divided into the two categories according to $\theta$, and the daily variation of $X, Y$, and $Z$ components at the five stations listed in Table 1 are averaged over each set of data. Figure 10 illustrates the results, where only the differences between positive and negative days are displayed. Hourly values during the 22 or 23 years are used in the analysis, but only the data on the day satisfying the following conditions are used throughout the procession: maximum $K p$ index in the day is not greater than $2+$; no missing value is included in data of any component; $\theta$ for the day is available; and the 'amplitude' of the oscillation, defined by Eq. (11), on the day is larger than a half of its standard deviation. The purpose of the last condition is to exclude the days when the oscillation was inactive because the calculation of $\theta$ is possibly inaccurate on those days. Before the composition, a trend on each day is subtracted by using hourly data in the nighttime interval.

Figure 10 indicates that the daily variation of each station has a clear dependence on the phase of the oscillation. The $X$ component is positive during the daytime in MMB and KAK, negative at GUA and PMG, while there is no distinctive variation at $\mathrm{CBI}$. The $Y$ component of the daily variation has a latitudinal profile almost symmetric with GUA. A negative peak in the morning is evident at each station in the northern hemisphere (MMB, KAK, and $\mathrm{CBI}$ ), while a positive peak is found at PMG in the southern hemisphere. A secondary peak having an opposite sense to the primary peak appears in the afternoon at PMG though the peak is ambiguous in the northern hemisphere. The symmetry with the equator is also seen in the daily variation of the $Z$ component. At KAK and CBI, downward variation is clear in the daytime though upward variation exists at PMG. It should be noted that the symmetry axis of the latitudinal profile is near GUA, which is just north of the geomagnetic equator, while the nearest station to the geographic equator is PMG. The dependence of the daily profiles on the geomagnetic equator also supports the idea that the currents responsible for the oscillation flow in the ionosphere.

We carried out composite analyses also for the 2-day and 3 -day oscillations by using respective phase angles, and similar correlations with the oscillations at CBI were obtained (not shown here). The coherence of the daily variation of these stations with the oscillations means that the oscillations are not local phenomena but have some spatial extent in both hemispheres.

\section{Discussion}

Our results show that the day-to-day variation in the range shorter than $\sim 10$ days is composed of the superposition of the 2-day, 3-day, and 5-6-day oscillations, whose variances are not much different from each other. The intensity of each oscillation fluctuates considerably, and there exist active intervals like 'burst events.' Although there have been reports on magnetic field variations at other observatories 

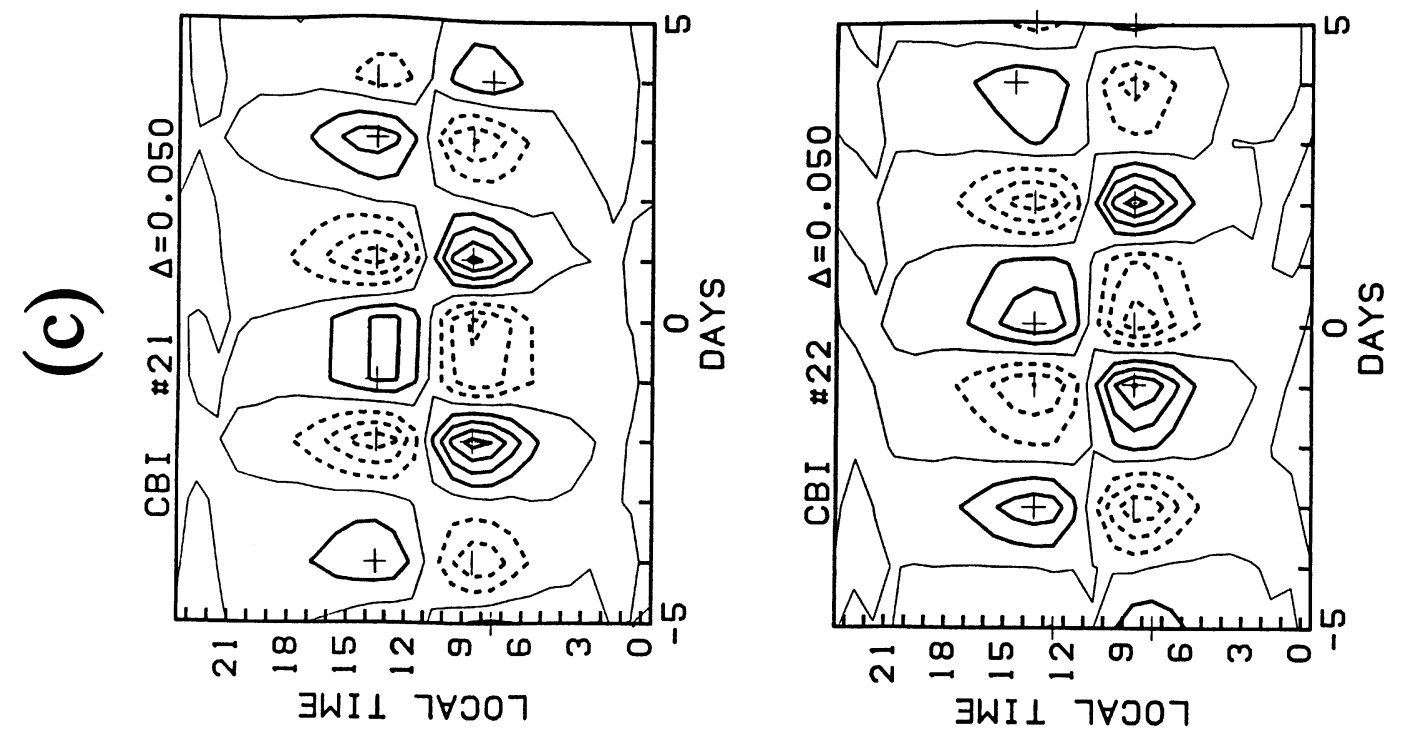

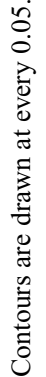
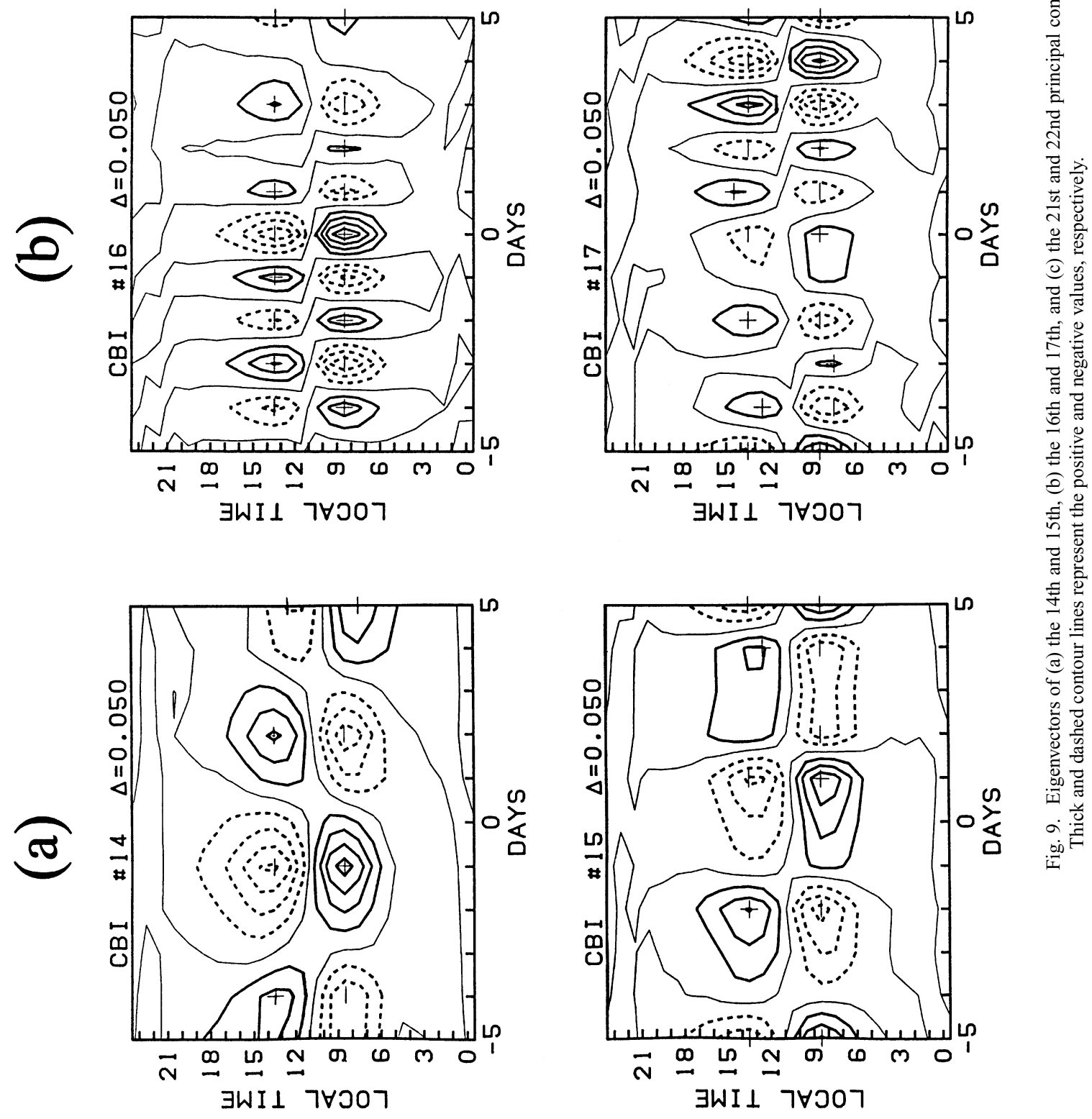


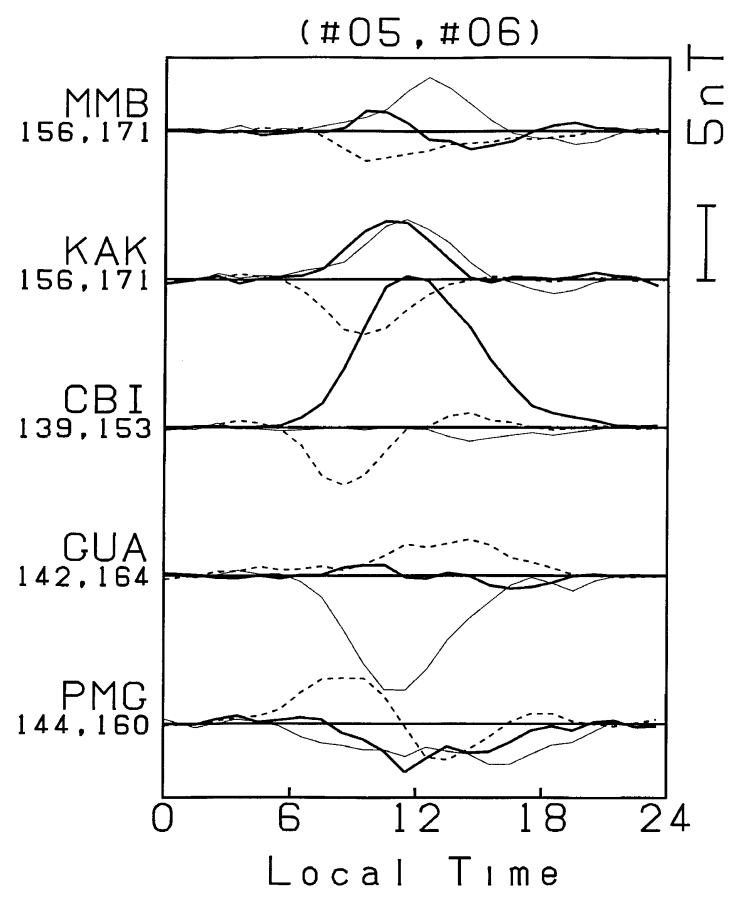

Fig. 10. Daily variations of the $X$ (thin line), $Y$ (dashed line), and $Z$ (thick line) components at five stations. Each line represents a composite daily profile correlating with the 5-6-day oscillation in the $Z$ component at Chichijima. Only the differences between positive and negative days of the midday anomaly of the oscillation are displayed. The two numerals beneath the name of each station are the numbers of days for positive and negative days, respectively.

showing spectral peaks in the vicinity of the periods (45 and 58 hours by Takeda and Yamada (1989), 3 days and 6 days by Parish et al. (1994)), the waveforms and those temporal variations of the oscillations were clarified by this analysis. The method used here is better able to extract and separate these oscillations having such characteristics than the spectral analysis usually used, because they are based on the assumption of the stationarity of the periodic variations. Moreover, the periods deduced by our method are more reliable because the existence of the periodic variations is not assumed throughout the analysis.

Each oscillation detected at CBI primarily consists of the day-to-day variation of the $S q$ amplitude. The perturbation is restricted almost entirely to the daytime, and is synchronously seen at least in a region stretching in both hemispheres. Therefore it is most likely that the oscillations are attributed to ionospheric currents. In particular, the 2-day oscillation is possibly brought about by the ionospheric dynamo currents driven by the atmospheric quasi 2-day wave, which is frequently observed in the mesosphere and lower thermosphere. Takeda and Yamada (1989) simulated the magnetic field variation on the ground using a wind model for the quasi 2-day wave (maximum wind velocity is $20 \mathrm{~m} / \mathrm{s}$ at $90 \mathrm{~km}$ altitude at $30^{\circ}$ latitude). Indeed their result showed that the ionospheric current is induced by the wind, but the intensity of the current system is only a few percent of that of $S q$ and the magnitude of the $Z$ component in low latitudes is less than $1 \mathrm{nT}$. This is in remarkable contrast to the result of our analysis: the peak value of the 2-day oscillation is larger than $3 \mathrm{nT}$ even in a typical case. It must be noted that, in their calculation of the ground magnetic field, the contribution from the currents induced in the earth was neglected. If the induced currents were included in the model, this discrepancy would be enlarged because amplitudes of the vertical component are generally reduced by the induced currents.

In the calculation of Takeda and Yamada (1989), the wind of the 2-day wave was assumed to blow only below 105 $\mathrm{km}$ altitude. It is most likely that the discrepancy noted above can be attributed to the contribution from the wind in higher atmosphere. Although the quasi 2-day wave in the ionosphere is not fully understood, the oscillation with the period has been found also in the electron density of the F-layer (Chen, 1992; Pancheva et al., 1994; Apostolov et al., 1995). This suggests the existence of the 2-day periodic motion at altitudes greater than $105 \mathrm{~km}$. It should be noted on the possibility that the spatial structure of the motion in the dynamo region associated with the 2-day oscillation is much different from that of the middle atmosphere. Apostolov et al. (1995) found two types of the quasi 2-day wave in the foF 2 fluctuations with zonal wavenumbers 1 and 0 . These wavenumbers are different from those observed in the middle atmosphere, wavenumber 3 or 4 (Glass et al., 1975; Rodgers and Prata, 1981; Meek et al., 1996). The existence of the broad spectral peak at $\sim 48$ hours in the global geomagnetic indices $a n, a s$, and am infers that the oscillation of the magnetic field is in-phase in longitudes (Apostolov et al., 1996). These results suggest that the velocity field above the middle thermosphere is much different from that at the lower altitude.

The 2-day oscillation of the magnetic field deduced here shows the amplitude modulation having a period of several days. While it may reflect the amplitude fluctuation of the responsible wind system, another explanation for the modulation is possible when the period of the oscillation of the atmosphere is slightly different from 48 hours. Even if the velocity field be globally distributed, the ionospheric currents would be considerably reduced in the nighttime because of the daily variation of ionospheric conductivity. Consequently, the magnetic signal produced by the wind system only in the daytime could be obtained on the ground. If the period of the wind is slightly different from 48 hours, which is evident for the quasi 2-day wave in the middle atmosphere (Tsuda et al., 1988), the magnitude of the daytime signature of the magnetic field would vary from day to day, with a longer period than 2 days when viewed at a fixed point on the ground.

It is more difficult to discuss, at present, the mechanism of the oscillations of the other two periods, 3-day and 5-6-day, found in the analysis. Since Parish et al. (1994) also found the 3-day and 6-day spectral peaks in the $H$ component at the geomagnetic equator, Huancayo, these oscillations we have extracted are probably global phenomena. There possibly exists 3-day and 5-6-day oscillations in the wind which drive the ionospheric dynamo currents. This idea is supported by the fact that the spectral peaks of 3-4 and 5-7 days (Luo and Chen, 1993) and of $\sim 5$ days (Apostolov et al., 1994) were detected in the foF 2 time series.

As discussed above we have only limited knowledge on the mechanism of the day-to-day variation of the geomag- 
netic field including the 2-day, 3-day, and 5-6-day oscillations. In the next step, the global structure of each oscillation of the magnetic field should be investigated to estimate the two-dimensional distribution of the ionospheric current driving the oscillation. This would also give us an important clue to infer the wind distribution in the dynamo region, where observations are difficult by other means, and would increase our knowledge of the dynamical couplings between the middle atmosphere and the ionosphere.

\section{Conclusions}

The day-to-day variation of the geomagnetic $Z$ component of Chichijima was investigated by a method based on principal component analysis. It was applied in such a way as to achieve greater understanding of the day-to-day variation of the waveform of $S q$ than ever used. The following results have been obtained:

(1) There exist 5-6-day, 3-day, and 2-day oscillations in the variation. The fluctuations associated with the oscillations are restricted almost entirely to the daytime, which suggests that the oscillations are due to ionospheric currents.

(2) The daily profile of each oscillation is similar to the mean daily variation. This means that these oscillations appear primarily in the amplitude of the daily variation.

(3) Although the oscillations seem to be present almost continuously, the amplitude of each oscillation is highly variable.

(4) In the case of typical activities, the magnitude of the fluctuation associated with the 2-day, 5-6-day, and 3-day oscillations amount to $3.3,2.8$, and $2.2 \mathrm{nT}$, respectively. These values are $17-12 \%$ of the range of the mean daily variation of the $Z$ component at Chichijima. There are cases, however, when the magnitude is considerably amplified during a short duration.

(5) The 2-day oscillation is accompanied by the amplitude modulation of the period of $\sim 10$ days. This is probably originated both from the difference in the period of the driving force from 48 hours and from the daily variation of the ionospheric conductivity. This also supports the idea that the oscillation is caused by the quasi-2-day wave in the ionosphere.

(6) The pattern of $S q$ as well as its intensity oscillates with the three periods. This suggests the existence of eastwest oscillations of the $S q$ focus in the daytime.

(7) Day-to-day variations at other four stations along almost the same meridian as Chichijima were investigated. The result indicates that the oscillations are phenomena having some latitudinal extent across the geomagnetic equator.

Acknowledgments. The author would like to thank Dr. M. Takeda for his valuable comments on this manuscript. The author also sincerely acknowledges Dr. H. Nagao and Dr. L. Wang for their constructive reviews, and Prof. Y. Ogawa for his helpful comments and suggestions. The Dst index and a part of geomagnetic data were provided by the World Data Center for Geomagnetism, Kyoto University.

\section{References}

Apostolov, E. M., L. Alberca, and D. Altadill, Solar cycle and seasonal behaviour of quasi-two- and five-day oscillations in the time variations of foF2, Annali di Geofisica, 37, 187-192, 1994.
Apostolov, E. M., D. Altadill, and L. Alberca, Characteristics of quasi2-day oscillations in the foF2 at northern middle latitudes, J. Geophys. Res., 100, 12163-12171, 1995.

Apostolov, E. M., D. Altadill, and L. Alberca, Persistence of quasi-2-day oscillations in the geomagnetic activity indices (an, as, am), J. Geomag. Geoelectr., 48, 1233-1239, 1996.

Chen, P. R., Two-day oscillation of the equatorial ionization anomaly, $J$. Geophys. Res., 97, 6343-6357, 1992.

Forbes, J. M. and S. Leveroni, Quasi 16-day oscillation in the ionosphere, Geophys. Res. Lett., 19, 981-984, 1992.

Glass, M., J. L. Fellows, M. Massebeuf, A. Spizzichino, I. A. Lysenko, and $\mathrm{Yu}$ I. Portniaghin, Comparison and interpretation of the results of simultaneous wind measurements in the lower thermosphere at Garchy (France) and Obninsk (U. S. S. R.) by meteor radar technique, J. Atmos. Terr. Phys., 37, 1077-1087, 1975.

Golovkov, V. P., N. Ye. Papitashvili, Yu. S. Tyupkin, and Ye. P. Kharin, Separation of geomagnetic field variations into quiet and disturbed components by the method of natural orthogonal components, Geomag. Aero., 18, 342-344, 1978.

Hasegawa, M., On the position of the focus of the geomagnetic $S q$ current system, J. Geophys. Res., 65, 1437-1447, 1960.

Ito, R., S. Kato, and T. Tsuda, Consideration of an ionospheric wind dynamo driven by a planetary wave with a two-day period, J. Atmos. Terr. Phys., 48, 1-13, 1986

Kohsiek, A., K. H. Glassmeier, and T. Hirooka, Periods of planetary waves in geomagnetic variation, Ann. Geophys., 13, 168-176, 1995.

Kohsiek, A., M. Kiefer, C. E. Meek, and A. H. Manson, Fluctuations in tides and geomagnetic variations, Geophys. Res. Lett., 25, 889-892, 1998.

Krzanowski, W. J., Principles of Multivariate Analysis, Revised ed., Oxford Univ. Press, New York, 2000.

Luo, Y. and P. R. Chen, Long period oscillations in the Equatorial Ionization Anomaly correlated with the neutral wind in the mesosphere, J. Atmos. Terr. Phys., 55, 1317-1323, 1993.

Meek, C. E., A. H. Manson, S. J. Franke, W. Singer, P. Hoffmann, R. R. Clark, T. Tsuda, T. Nakamura, M. Tsutsumi, M. Hagan, D. C. Fritts, J Isler, and Yu I. Portnyagin, Global study of northern hemisphere quasi2-day wave events in recent summers near $90 \mathrm{~km}$ altitude, J. Atmos. Terr Phys., 58, 1401-1411, 1996.

Pancheva, D., L. F. Alberca, and B. A. de la Morena, Simultaneous observation of the quasi-two-day variations in the lower and upper ionosphere over Europe, J. Atmos. Terr. Phys., 56, 43-50, 1994.

Parish, H. F., J. M. Forbes, and F. Kamalabadi, Planetary wave and solar emission signatures in the equatorial electrojet, J. Geophys. Res., 99, 355-368, 1994.

Parkinson, W. D., Bi-diurnal geomagnetic variations, Ann. Geophys., 38, 327-329, 1982.

Philips, A. and B. H. Briggs, The day-to-day variability of upper atmosphere tidal winds and dynamo currents, J. Atmos. Terr. Phys., 53, 39-47, 1991.

Press, W. H., S. A. Teukolsky, W. T. Vetterling, and B. P. Flannery, Numerical Recipes in FORTRAN, The Art of Scientific Computing, 2nd ed., Cambridge Univ. Press, Cambridge, 1992.

Rajaram, M., Method of natural orthogonal components applied to equatorial geomagnetic variations, Annls. Géophys., 36, 599-603, 1980.

Rodgers, C. D. and A. J. Prata, Evidence for a traveling two-day wave in the middle atmosphere, J. Geophys. Res., 86, 9661-9664, 1981.

Suzuki, A., UT and day-to-day variations in equivalent current systems for world geomagnetic variations, J. Geomag. Geoelectr., 31, 21-46, 1979.

Takeda, M. and Y. Yamada, Quasi two-day period variation of the geomagnetic field, J. Geomag. Geoelectr., 41, 469-478, 1989.

Tsuda, T., S. Kato, and R. A. Vincent, Long period wind oscillations observed by the Kyoto meteor radar and comparison of the quasi-2-day wave with Adelaide HF radar observations, J. Atmos. Terr. Phys., 50, 225-230, 1988.

Vertlib, A. B. and C.-U. Wagner, Analysis of geomagnetic $S q$ variations by the expansion of fields in natural orthogonal components, 1. Method and problems, Geomag. Aero., 10, 509-513, 1970.

World Data Center for Geomagnetism, Kyoto, DATA CATALOGUE, No. 25, Kyoto Univ., 1999.

$\mathrm{Xu}, \mathrm{W} .-\mathrm{Y} ., \mathrm{On} S q$ and $L$ current systems in the ionosphere, PAGEOPH, 131, 515-525, 1989.

Y. Yamada (e-mail: ymd@kakioka-jma.go.jp) 